\title{
TERAPIA FOTODINÁMICA EN DISTROFIA FOVEOMACULAR VITELIFORME DEL ADULTO
}

\section{PHOTODYNAMIC THERAPY IN ADULT-ONSET FOVEOMACULAR VITELLIFORM DISTROPHY}

\author{
ABENGOECHEA-HERNÁNDEZ $\mathrm{S}^{1}$, ELIZALDE-MONTAGUT $\mathrm{J}^{2}$, FIDELIZ DE LA PAZ-DALISAY M ${ }^{3}$
}

\begin{abstract}
RESUMEN
Caso clínico: Se describe el caso de un paciente al que se le realizó terapia fotodinámica con Verteporfin (TFD) en una distrofia foveomacular viteliforme del adulto (DFVA) en otro servicio al ser confundido con una membrana neovascular coroidea. La tomografía de coherencia óptica (OCT) es de gran ayuda en el diagnóstico diferencial. El paciente sufrió una disminución de la agudeza visual (AV) severa a los dos años del tratamiento con signos angiográficos de hipoperfusión coroidea.

Discusión: La DFVA es una entidad poco frecuente caracterizada por tener un buen pronóstico visual si bien existen casos con limitación de la AV severa en cortos períodos de tiempo en su historia natural. La OCT presenta una imagen claramente diferente respecto a la neovascularización coroidea. La terapia fotodinámica con Verteporfin puede no tener un efecto positivo a largo plazo en pacientes con DFVA.
\end{abstract}

Palabras clave: Terapia fotodinámica, distrofia foveomacular viteliforme del adulto, tomografía de coherencia óptica, degeneración macular asociada a la edad, membrana neovascular coroidea.

\begin{abstract}
Case report: We present the case of a patient who underwent photodynamic therapy (PDT) for adultonset foveomacular vitelliform dystrophy (AFVD) at another clinic, having been misdiagnosed as having a choroidal neovascular membrane. Two years post-treatment, the patient had severe central visual loss and showed angiographic signs of choroidal hypoperfusion.

Discussion: AFVD is a rare clinical entity, usually having a fair visual prognosis, although some cases may show episodes of severe visual loss during their clinical course. Optical coherence tomography (OCT) is valuable in revealing the distinct images of AFVD from the features of a choroidal neovascular membrane. PDT using verteporfin may have negative long term effects if used in patients with AFVD (Arch Soc Esp Oftalmol 2007; 82: 117-120).
\end{abstract}

Key words: Photodynamic therapy, adult-onset foveomacular vitelliform dystrophy, optical coherence tomography, age-related macular degeneration, choroidal neovascular membrane.

Recibido: 5/5/06. Aceptado: 13/2/07.

Centro de Oftalmología Barraquer. Barcelona. España.

1 Licenciado en Medicina. Departamento de Vítreo-Retina.

2 Doctor en Medicina. Departamento de Vítreo-Retina.

3 Licenciada en Medicina. Departamento de Segmento Anterior.

Correspondencia:

Santiago Abengoechea Hernández

C/. Muntaner, 314

08021 Barcelona

España

E-mail: sah@co-barraquer.es 


\section{INTRODUCCIÓN}

La distrofia foveomacular viteliforme del adulto (DFVA) es una enfermedad poco frecuente con un patrón de herencia autosómico dominante. Se caracteriza por presentar una disminución de visión moderada en la edad adulta y tener un buen pronóstico en la mayoría de casos. La afectación es bilateral si bien se han descrito casos unilaterales. El electrooculograma suele ser normal o levemente alterado. Biomicroscópicamente se caracteriza por presentar un depósito amarillento subretiniano localizado en el área macular. Esta entidad presenta una imagen angiográfica que puede ser confundida con una neovascularización coroidea. La tomografía de

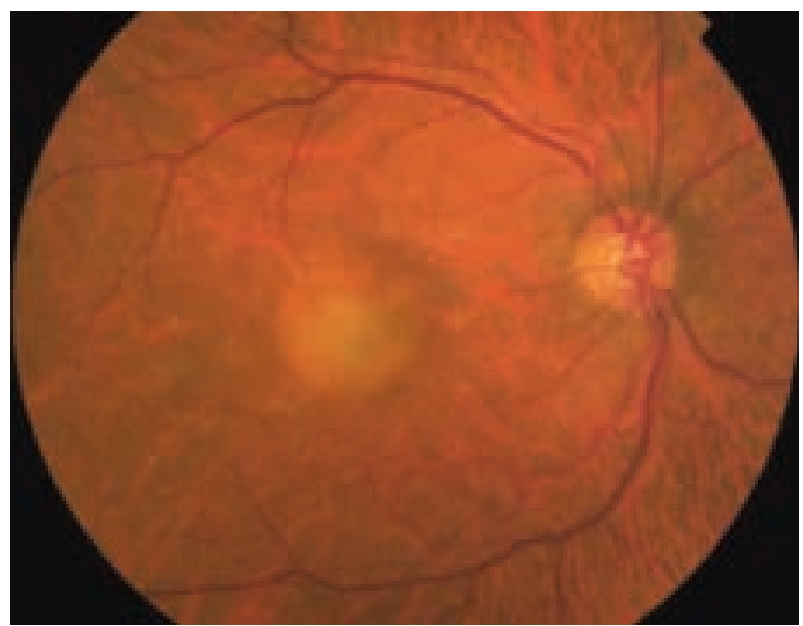

Fig. 1: Lesión viteliforme subfoveal. Drusas duras en polo posterior. coherencia óptica (OCT) es de gran ayuda en el diagnóstico diferencial.

\section{CASO CLÍNICO}

Varón de 65 años de edad con antecedentes de dos sesiones de terapia fotodinámica con Verteporfin (TFD) y propuesto para una tercera acude para valoración. Su agudeza visual actual es de 0,7 en ojo derecho (OD). Se trata de un ojo único ya que el ojo izquierdo (OI) está en ptisis bulbi debido a un antecedente traumático ocurrido en su niñez. El paciente no refiere cambios desde el inicio del cuadro y tampoco había notado variación de su agudeza visual antes de haberle realizado las sesiones de TFD. La imagen funduscópica revela una lesión viteliforme sobreelevada en área foveal con drusas duras en el polo posterior (fig. 1). En la angiografía fluoresceínica (AGF) se aprecia una hiperfluorescencia en anillo progresiva e intensa en tiempos tardíos con una zona central hipofluorescente (figs. 2a y 2b). En la angiografía con verde de indocianina (AVI) no se aprecian alteraciones de interés. En la OCT se observa la presencia de una estructura cuneiforme de reflectividad media-alta entre el epitelio pigmentado y la retina neurosensorial con un desprendimiento de la retina neurosensorial bordeando la lesión (fig. 3). Se le realizó un electrooculograma siendo su resultado dentro de los límites de la normalidad.

El paciente es diagnosticado de DFVA y se decide realizar controles periódicos. En los controles de

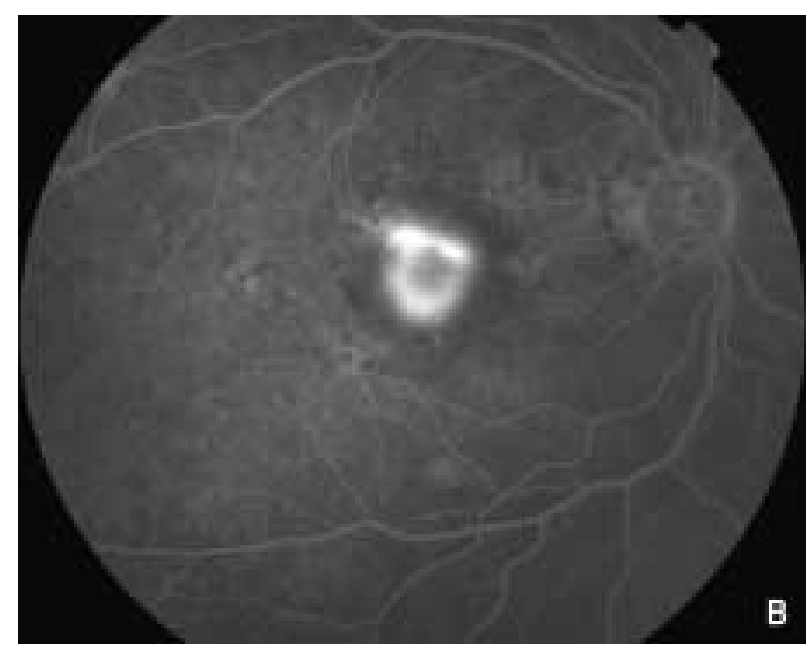

Fig. 2: Hiperfluorescencia en anillo que aumenta con el tiempo angiográfico con un área de hipofluorescencia central. 


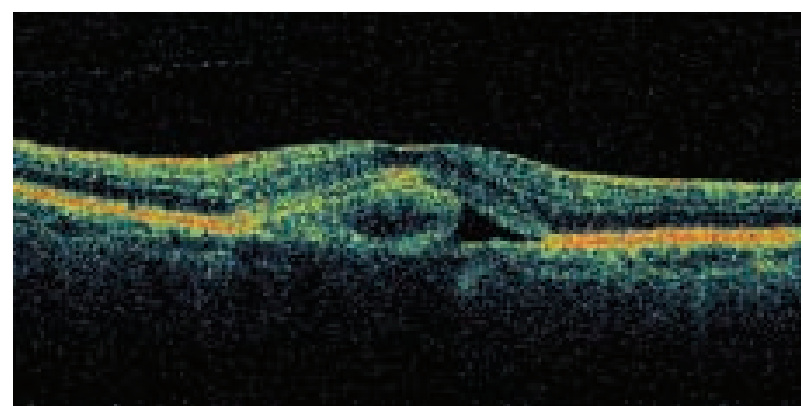

Fig. 3: Lesión de reflectividad media localizada entre el epitelio pigmentado y la retina. Existe un levantamiento neurosensorial en cuña junto al material subfoveal.

los 3, 6 y 12 meses los resultados de las pruebas no muestran variación respecto a la primera visita y su agudeza visual permanece estable.

En el control de los 24 meses su agudeza visual es de 0.02. En el fondo de ojo no se observa la lesión viteliforme sino una lesión redondeada anaranjada en el área foveal (fig. 4). Tanto en la AGF como en la AVI existe una hipofluorescencia intensa mantenida en todo el tiempo angiográfico (figs. $5 a, 5 b$ y $5 c)$. En la OCT se aprecia una depresión foveal normal con ausencia de la masa de reflectividad media apreciada en la primera visita y un aumento de la reflectividad posterior a la altura del complejo epitelio pigmentado-coriocapilar (fig. 6).

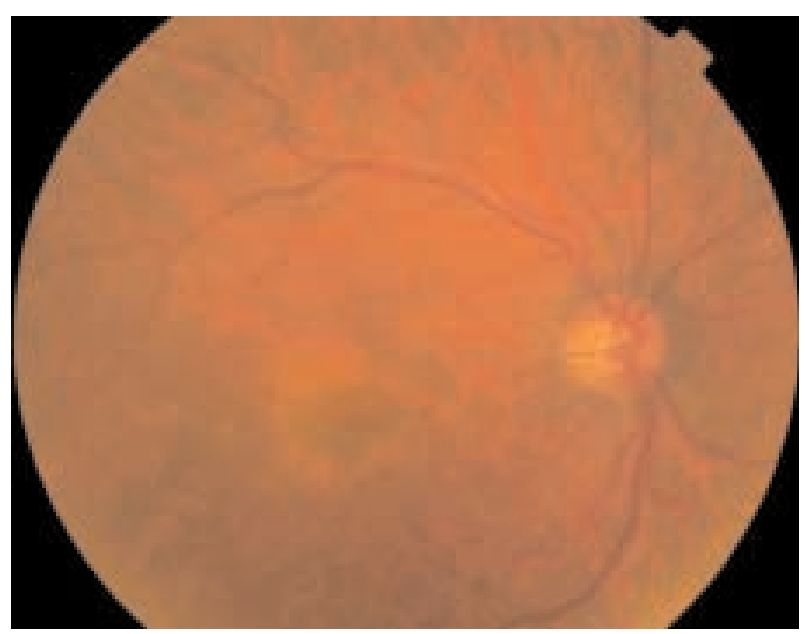

Fig. 4: Lesión redondeada anaranjada en área foveal donde estaba localizada la estructura viteliforme.
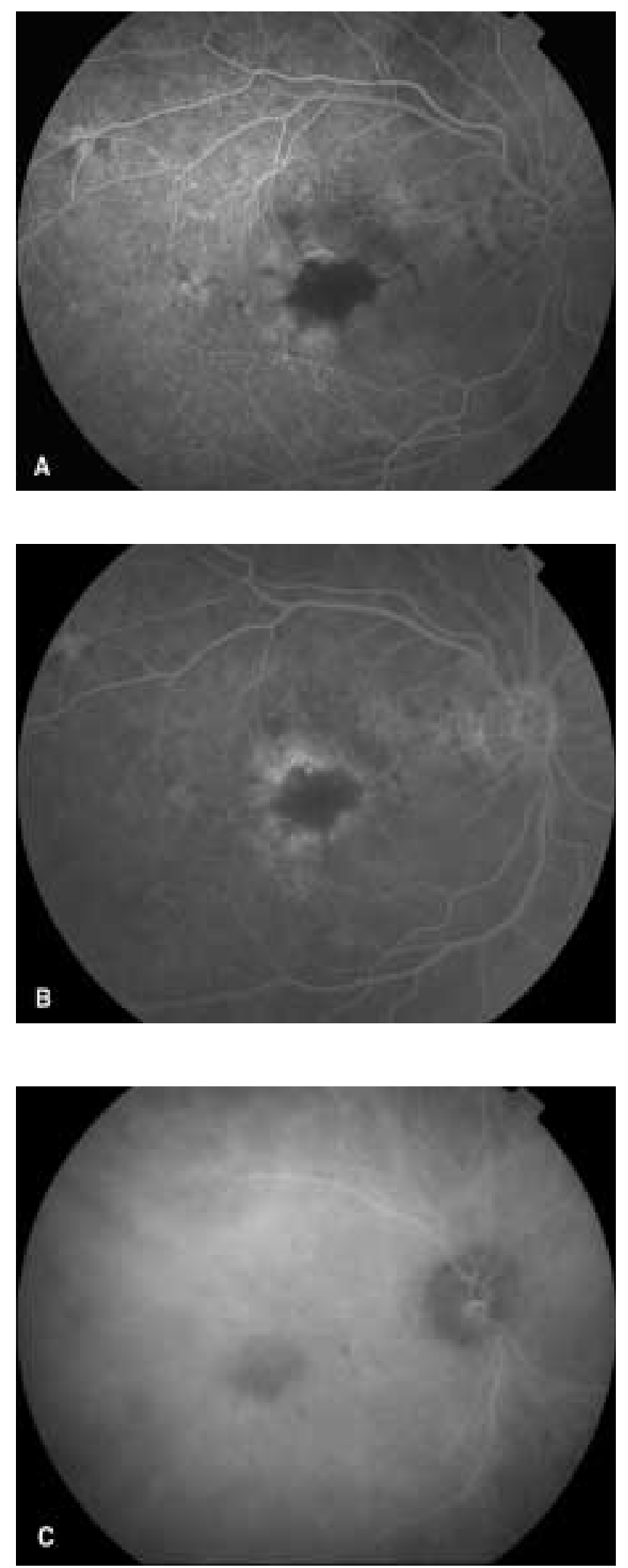

Fig. 5: Hipofluorescencia mantenida en todo el tiempo angiográfico por hipoperfusión coroidea. 


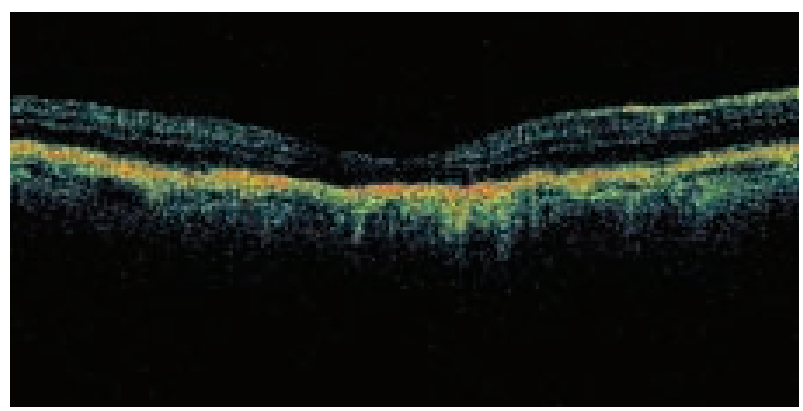

Fig. 6: Depresión foveal conservada con aumento de la reflectividad posterior. Ausencia del material localizado entre el epitelio pigmentado y la retina neurosensorial.

\section{DISCUSIÓN}

El comportamiento angiográfico de la primera visita puede hacer pensar en la existencia de una membrana neovascular subfoveal debido a la intensa hiperfluorescencia en tiempos tardíos pero el patrón de la OCT es muy característico de la DFVA donde el levantamiento neurosensorial podría estar ocasionado por la limitación funcional del epitelio pigmentado debido a la presencia de la lesión viteliforme.

La OCT aporta una imagen claramente diferenciadora entre la DFVA y la neovascularización coroidea $(1,2)$. La ausencia de desprendimiento del epitelio pigmentado y signos de actividad neovascular junto con la presencia del material de reflectividad media-alta por debajo de la retina neurosensorial nos hace pensar en una DFVA frente a una membrana neovascular. Asimismo, la ausencia de una estructura de reflectividad alta entre el epitelio pigmentado y la retina neurosensorial resultante de la fibrosis de la supuesta membrana neovascular coroidea a los dos años acaba por confirmar el diagnóstico.

La presencia de una severa disminución de visión con hipofluorescencia intensa angiográfica y con ausencia del material viteliforme por OCT sugiere una hipoperfusión coroidea. Es difícil saber el gra- do de responsabilidad de la TFD en este cuadro ya que la merma visual se detecta a los 2 años de haber realizado la segunda sesión de tratamiento. Existen referencias bibliográficas describendo la reabsorción del material subretiniano tras la TFD y sin disminución de la agudeza visual (3). La historia natural de esta enfermedad también señala la posibilidad de disminución de la agudeza visual en corto espacio de tiempo y no de manera paulatina como es más frecuente observar en esta entidad (4). Existen autores que refieren la inocuidad del tratamiento aunque sus series únicamente llegan al año de seguimiento. En nuestro caso al año no presentaba ningún cambio respecto a la primera visita. Existen series más extensas y de seguimiento superior donde se ha podido evidenciar una pérdida severa de la agudeza visual por hipoperfusión coroidea (5).

Así pues, debemos conocer claramente el comportamiento angiográfico y tomográfico de la DFVA ya que confundirla con una neovascularización coroidea y realizar tratamiento con TFD podría eventualmente provocar una disminución severa e irreversible de la agudeza visual.

\section{BIBLIOGRAFÍA}

1. Pierro L, Tremolada G, Introini $U$, Calori $G$, Brancato $R$. Optical coherence tomography findings in adult-onset foveomacular vitelliform dystrophy. Am J Ophthalmol 2002; 134: 675-680.

2. Benhamou N, Souied EH, Zolf R, Coscas F, Coscas G, Soubrane $G$. Adult-onset foveomacular vitelliform distrophy: a study by optical coherence tomography. Am J Ophthalmol 2003; 135: 362-367.

3. Menchini U, Giacomelli G, Cappelli S, Giansanti F, Romani A, Virgili G. Photodynamic therapy in adult-onset vitelliform macular dystrophy misdiagnosed as choroidal neovascularization. Arch Ophthalmol 2002; 120: 17611763.

4. Hodes BL, Feiner LA, Sherman SH, Cunningham D. Progression of pseudovitelliform macular dystrophy. Arch Ophthalmol 1984; 102: 381-383.

5. Ergun E, Costa D, Slakter J, Yanuzzi LA, Stur M. Photodynamic therapy and vitelliform lesions. Retina 2004; 24: $399-406$ 